\title{
Inseguridad alimentaria en el área de influencia del Centro de Medicina Familiar y Comunitaria "San Pantaleón" (Provincia de Buenos Aires)
}

\section{Food insecurity in the area of influence of the Center of Family and Community Medicine "San Pantaleón" (Province of Buenos Aires) \\ Insegurança alimentar na área de influência do Centro de Medicina de Família e Comunidade "San Pantaleón" (Província de Buenos Aires)}

Gabriel Bulgach ${ }^{1}$, Fernando Vázquez Peña ${ }^{2}$, Carolina Carrara ${ }^{3}$, Karin Kopitowskit ${ }^{4}$ El presente artículo busca determinar cuan frecuente es la inseguridad alimentaria en la población de una zona del conurbano bonaerense. Para medirlo se utilizó un instrumento que es la Escala
de la Inseguridad Alimentaria basada en la Experiencia. Se pudo determinar que esta herramienta es válida para hacerlo y se detectó una inseguridad alimentaria moderada/severa de $47,29 \%$ y
severa de 6,57\%. Además se observó que la presencia de inseguridad alimentaria moderada/severa era más probable en los hogares con más de 4 integrantes y en aquellos con una jefatura de hogar femenina.

\section{Conceptos clave:} ¿Qué se sabe sobre el tema?

La inseguridad alimentaria es un problema en aumento en nuestro país y más aún en población vulnerable.

Existen diferentes instrumentos para medir la inseguridad alimentaria.

\section{¿Qué aporta este trabajo?}

Aporta la validación estadística en Argentina de la escala FIES recomendada por FAO

Permite determinar la prevalencia de la inseguridad alimentaria en nuestra población a cargo.

La inseguridad alimentaria se asocia, en nuestra muestra, con la jefatura de hogar femenina y con la presencia de 4 convivientes o más en el hogar.

1- Universidad de Buenos Aires. Facultad de Ciencias Sociales; Argentina. ORCID: https://orcid.org/0000-0002-92709791. E-mail de contacto: gbulgach@gmail.com

2- Dr. en Medicina de la Universidad de Buenos Aires. Medicina Familiar y Comunitaria. Hospital Italiano de Buenos Aires; Argentina. ORCID: https://orcid.org/0000-0002-0214-6949. E-mail de contacto: fernandoramon.vazquez@hospitalitaliano.org.ar

3- Magtr en Efectividad Clínica. Servicio de Medicina Familiar y Comunitaria del Hospital Italiano de Buenos Aires; Argentina. ORCID: https://orcid org/0000-0002-3176-7971. E-mail de contacto: carolina.carrara@hospitalitaliano.org.ar.

4- Servicio de medicina Familiar y Comunitaria. Hospital Italiano de Buenos Aires; Argentina. Dir. Departamento de Investigación. Instituto Universitario Hospital Italiano de Buenos Aires; Argentina. ORCID: https://orcid.org/0000-0003-0939-0263. Email de contacto: karin.kopitowski@hospitalitaliano.org.ar

\section{Resumen:}

Introducción: La inseguridad alimentaria es una problemática en aumento en los últimos años en Argentina y resulta un indicador clave del estado de salud de la población. Este estudio se propuso determinar su prevalencia en una población del conurbano bonaerense planteándose como objetivos secundarios: 1) evaluar variables que se asocien con la inseguridad alimentaria; 2 ) realizar la validación estadística provisional de la Escala de la Inseguridad Alimentaria basada en la Experiencia (FIES). Materiales y métodos: Se realizó un estudio de investigación descriptivo, de corte transversal. Se utilizó la encuesta FIES en personas mayores de 15 años del Barrio Santa María en el Bajo Boulogne, partido de San Isidro, provincia de Buenos Aires. Se realizó un muestreo probabilístico. Se analizaron los datos siguiendo el modelo Rasch propuesto por la Organización de las Naciones Unidas para la Alimentación y la Agricultura. Resultados: Se entrevistaron 222 personas hallando una prevalencia de inseguridad alimentaria moderada/severa de $47,29 \%$ y severa de $6,57 \%$. Se observó una asociación independiente con una jefatura de hogar femenina OR 2,22; (IC95\% 1,27 a 3,87) y con tener más de 4 convivientes en el hogar OR 2,24; (IC95\% 1,25 a 4,02). Se realizó la validación provisional de la encuesta FIES en nuestra muestra a través del testeo de las asunciones del modelo Rasch. Conclusión: la inseguridad alimentaria es un problema presente en nuestra población. Se realizó la validación de un instrumento para medirla, de sencilla aplicación y que permite realizar comparaciones válidas entre mediciones locales e internacionales.

Palabras clave: seguridad alimentaria; Argentina; adultos; psicometría.

\section{Abstract:}

Introduction: Last years, food insecurity is a raising problem in Argentina and is a key indicator of population health. The principal aim of this study was to determine food insecurity prevalence in a population of Buenos Aires suburbs, considering as secondary objectives: 1) to evaluate associated variables with food insecurity; 2) to perform provisional statistical validation of the Food Insecurity Experience Scale (FIES). Methods: It was conducted a descriptive, cross-sectional research study was conducted. Check the FIES survey in people over 15 years of age in the Barrio Santa María in Bajo Boulogne, San Isidro district, Buenos Aires. A probabilistic sampling was carried out. The data will be analyzed following the Rasch model proposed by the Food and Agriculture Organization of the United Nations. Results: 222 people were interviewed, finding a prevalence of moderate / severe food insecurity of $47.29 \%$ and severe of $6.57 \%$. We found an independent association with a female head of household OR $2.22 ;(95 \% \mathrm{Cl}$ 1.27 to 3.87$)$ and having more than 4 cohabitants in the home OR $2.24 ;(95 \% \mathrm{Cl} 1.25$ to 4.02). The provisional validation of the FIES survey was carried out in our sample through the testing of the Rasch model assumptions. Conclusion: food insecurity is a problem present in our population. It was carried out the validation of an instrument to measure it, easy to apply and that allows valid comparisons between local and international measurements.

Keywords: food supply; Argentina; adult; psychometrics

\section{Resumo:}

Introdução: A insegurança alimentar é um problema crescente nos últimos anos na Argentina e é um indicador-chave do estado de saúde da população. Este estudo teve como objetivo determinar sua prevalência em uma população dos subúrbios de Buenos Aires, considerando como objetivos secundários: 1) avaliar variáveis associadas à insegurança alimentar; 2) realizar a validação estatística provisória da Escala de Vivência da Insegurança Alimentar (FIES). Materiais e métodos: Foi realizado um estudo descritivo, de corte transversal. A pesquisa FIES foi usada em pessoas com mais de 15 anos de idade no bairro Santa María em Bajo Boulogne, distrito de San Isidro, província de Buenos Aires. Uma amostragem probabilística foi realizada. Os dados foram analisados de acordo com o modelo Rasch proposto pela Organização para Alimentação e Agricultura das Nações Unidas. Resultados: 222 pessoas foram entrevistadas, encontrando-se uma prevalência de insegurança alimentar moderada / grave de 47,29\% e grave de $6,57 \%$. Encontrou-se uma associação independente se o chefe da família era uma mulher OR 2,22; (IC 95\% 1,27 a 3,87) e com mais de 4 coabitantes em casa OR 2,24; (IC 95\% 1,25 a 4,02). A validação provisória da pesquisa FIES foi realizada em nossa amostra através do teste das premissas do modelo Rasch. Conclusão: A insegurança alimentar é um problema presente em nossa população. Foi realizada a validação de um instrumento para mensuração, de fácil aplicação e permitindo comparações válidas entre medições locais e internacionais. 


\section{INTRODUCCIÓN}

Toda persona tiene derecho a una alimentación adecuada, que comprende aspectos cuantitativos, cualitativos y de aceptabilidad cultural, y el derecho fundamental a no padecer hambre, según las normas internacionales de derechos humanos ${ }^{(1)}$.

En el año 2009, en la Declaración de la Cumbre Mundial sobre Seguridad Alimentaria, se acordó la definición de la inseguridad alimentaria, planteando que "existe seguridad alimentaria cuando todas las personas tienen en todo momento acceso físico, social y económico a suficientes alimentos inocuos y nutritivos para satisfacer sus necesidades alimenticias y sus preferencias en cuanto a los alimentos a fin de llevar una vida activa y sana"(2). Este concepto incluye cuatro dimensiones (disponibilidad, acceso, utilización y estabilidad) ${ }^{(3)}$.

La disponibilidad aborda la cuestión de la "oferta", y es función del nivel de producción de alimentos, los niveles de existencias y el comercio neto. Para esta dimensión, la variable de mayor impacto es la producción.

La dimensión de acceso se refiere a la "demanda" y es función de los ingresos, del nivel de precios, así como de la presencia y cobertura de redes de protección social. Por lo que tiene una fuerte correlación con los niveles de ingresos y con las condiciones socioeconómicas. Pero también a la composición de los hogares, el lugar y características de la residencia, condición de la jefatura de hogar, niveles educativos alcanzados, condiciones sanitarias de las viviendas y las diversas variables que afectan y develan las condiciones de existencia.

La dimensión de utilización se refiere a la utilización biológica de los alimentos, dependiente principalmente del estado de salud de las personas, para lograr un adecuado bienestar nutricional que satisfaga las necesidades fisiológicas, asociándose a los indicadores de nutrición. La higiene y el saneamiento, la calidad del agua, las prácticas de cuidado de la salud, la comensalidad, así como la calidad e inocuidad de los alimentos, son elementos que determinan el buen aprovechamiento de los alimentos por parte del cuerpo.

Desde el siglo XIX, Argentina es uno de los mayores productores de alimentos del mundo. Según el INTA, la disponibilidad interna del conjunto de alimentos per cápita en Argentina creció un $78 \%$ en los últimos cincuenta años (1963-2013). Partiendo de 1.127 $\mathrm{kg} /$ persona/año para todos los alimentos considerados, se alcanzan una DIPC de $2.003 \mathrm{~kg} /$ persona/año respectivamente al final del período ${ }^{(4)}$. La problemática de la seguridad alimentaria en Argentina, se centra en las dimensiones de acceso y de utilización.

La cuestión alimentaria emergió como una de las problemáticas sociales más fuertes durante el 2019 en nuestro país. En su edición de ese mismo año la Organización de las Naciones Unidas para la Alimentación y la Agricultura (FAO) consigna que, para Argentina, la prevalencia de la inseguridad alimentaria grave pasó del $5,8 \%$ en 2014-2016 al $11,3 \%$ en 2016-2018, mientras que la inseguridad alimentaria moderada/ grave pasó del $19,1 \%$ en $2014-2016$ al $32,1 \%$ en $2016-2018^{(5)}$.

El estudio de Rosso y col realizado en la ciudad de Santa Fe en 2011, halló que el $31,5 \%$ de los hogares presentaba inseguridad alimentaria, de los cuales el $21,7 \%$ era leve, el $7,4 \%$ moderada y el $2,4 \%$ severa $^{(6)}$. En otro estudio desarrollado por el Observatorio de la Deuda Social, se afirma que el $11,2 \%$ de hogares con residencia en los principales centros urbanos del país experimentó algún grado de inseguridad alimentaria. De este total, el 6,6\% de los hogares presentaba inseguridad alimentaria moderada y el $4,6 \%$ severa $^{(7)}$.

El Servicio de Medicina Familiar y Comunitaria del Hospital Italiano en su Centro de Medicina Familiar y Comunitaria "San Pantaleón" (CMFyCSP) asiste a un amplio sector de la población del barrio Bajo Boulogne, municipio de San Isidro, en la provincia de Buenos Aires.

En el inicio del año 2019 observamos situaciones que nos llevaron a cuestionarnos acerca de la situación alimentaria de la población, como el aumento de demanda de comida al centro de salud, escuelas, y sede de Acción Social del municipio, sumado a la aparición y aumento en la cantidad de comedores y merenderos. En ese contexto, conocer el nivel de inseguridad alimentaria resulta trascendente como un indicador clave del estado de salud de la población.

El objetivo del presente estudio fue determinar la prevalencia de inseguridad alimentaria de la población del área de influencia del CMFyCSP. Se planteó como objetivos secundarios: 1) evaluar variables que se asocien con la inseguridad alimentaria; 2 ) realizar la validación estadística provisional de la FIES.

\section{MATERIALES Y MÉTODOS}

Se realizó un estudio descriptivo, de corte transversal, con datos obtenidos de fuentes primarias. Se aplicó la Escala de la Inseguridad Alimentaria basada en la Experiencia, conocida por su acrónimo en inglés: FIES, siguiendo las instrucciones metodológicas para su aplicación ${ }^{(8-10)}$ a personas mayores de 15 años del Barrio Santa María en el Bajo Boulogne, partido de San Isidro, provincia de Buenos Aires que corresponde al área de influencia del CMFyCSP. Se realizó un muestreo probabilístico, seleccionando aleatoriamente, las manzanas del barrio, de un total de 56 manzanas, y luego, también en forma aleatoria, una calle de cada manzana, visitando todas las casas de cada calle seleccionada. En caso de que nadie respondiera se reiteraba la visita. Ante la respuesta, se preguntaba cuántos convivientes había, dentro de la casa mayores de 15 años, indicándole que se seleccionaría aleatoriamente al entrevistado entre los dispuestos a contestar

\section{Instrumento}

Se utilizó la encuesta FIES, que mide el acceso de las personas a los alimentos en cantidad y calidad suficientes en forma individual o en el hogar; y proporciona el conjunto de instrumentos necesarios para calcular indicadores válidos y fiables de prevalencia de la inseguridad alimentaria en diferentes niveles de gravedad ${ }^{(11)}$. Está compuesta por ocho preguntas con respuestas dicotómicas (sí/no). Sus ítems componen una escala estadística diseñada para cubrir un rango de la severidad de la inseguridad alimentaria y deben analizarse en conjunto, no como ítems separados. Se utilizó la versión para individuos con un período de referencia de 12 meses a fin de evitar la influencia de los efectos estacionales y mejorar la comparabilidad entre países (Ver anexo). Esta versión en idioma español es utilizada en nuestro país por la Encuesta Mundial de Gallup $\AA^{(12)}$

Los datos de la encuesta fueron recolectados por entrevistadores, que recibieron capacitación y contaron con un Manual para el entrevistador como medio de preparación previa y consulta durante la recolección de datos.

Ademá se recolectaron datos sociodemográficos.

\section{Tamaño muestral}

Se siguió la recomendación de los autores que indican que para una validación provisional es necesario un tamaño muestral entre 100 y 300 casos con scores intermedios (ni 0 , ni 8) $)^{(10)}$.

\section{Análisis de los datos}

Se validaron los resultados utilizando un modelo Rasch, siguiendo las instrucciones ya referidas. Se verificó que los ítems presentaran valores de Infit (verifican que el ítem evaluado funcione correctamente en la población estudiada), y Outfit (revelan la ausencia de valores atípicos) entre 0,7 y $1,3^{\left({ }^{(10)}\right.}$. Para este análisis se utilizó el programa STATA versión 13 que trabaja con valores estandarizados de Infit y Outfit ${ }^{(13)}$. Los valores equivalentes estandarizados, a los ya señalados estimados, son -2 y 2 respectivamente ${ }^{(14)}$.

Se evaluó la fiabilidad del modelo con el coeficiente KuderRicharson (KR-20), y con el valor de Fiabilidad compuesta, a través de Análisis Factorial Confirmatorio, realizado con el programa Lisrel 10.20. Considerándose adecuado un valor de fiabilidad mayor a $0,7^{(10)}$. 
Se utilizó la calculadora Excel provista por $\mathrm{FAO}^{(15)}$. Se estimaron, para cada ítem, la diferencia absoluta entre la severidad estándar (global, internacional) luego de la calibración (equiparación de la media y el desvío estándar del conjunto de elementos, en una métrica común), y la severidad del ítem en nuestra muestra. Para calcular la prevalencia de inseguridad alimentaria, se determinó como ítems "únicos" aquellos cuya diferencia absoluta es importante entre las escalas que se comparan, e ítems "comunes" a aquellos que presentan diferencias absolutas menores, son comparables, y por lo tanto se introducen sus valores en la ecuación. También se consideró, para decidir cómo clasificar en este sentido a los 8 elementos, la correlación entre los ítems "comunes"(10).

Con el fin de valorar la asociación de ciertas variables con la inseguridad alimentaria moderada/severa se realizó un análisis bivariado en el que se identificaron variables con las que luego se construyó un modelo de regresión logística.
Aspectos éticos del proyecto

El protocolo fue aprobado por el Comité de Ética de Protocolos de Investigación del Hospital Italiano de Buenos Aires y cumple con la Ley de Investigación de la Provincia de Buenos Aires $11044^{(16)}$ y Resolución № 1480/2011. Guía para Investigaciones con Seres Humanos. Ministerio de Salud de la Nación ${ }^{(17)}$.

Se solicitó consentimiento oral a todos los participantes.

\section{RESULTADOS}

Se realizaron 222 encuestas en 28 manzanas aleatorizadas del área de influencia del CMFyCSP. Las características sociodemográficas de las personas entrevistadas se observan en la Tabla 1.

Tabla $\mathbf{N}^{\circ}$ 1: Características sociodemográficas de las personas entrevistadas

\begin{tabular}{|c|c|}
\hline Características sociodemográficas & Total=222 \\
\hline Mujeres, N (\%) & $154(69,68)$ \\
\hline Edad, media $\pm \mathrm{DE}$ & $47,23 \pm 17,57$ \\
\hline \multicolumn{2}{|l|}{ Cantidad de personas en el hogar, $\mathrm{N}(\%)$} \\
\hline 0 & $2(0,9)$ \\
\hline 1 & $19(8,6)$ \\
\hline 2 & $38(17,1)$ \\
\hline 3 & $37(16,7)$ \\
\hline 4 & $50(22,5)$ \\
\hline 5 & $34(15,3)$ \\
\hline 6 & $25(11,3)$ \\
\hline 7 & $7(3,2)$ \\
\hline 8 & $6(2,7)$ \\
\hline 9 & $3(1,4)$ \\
\hline 12 & $1(0,5)$ \\
\hline Presencia de menores de 15 años, $\mathrm{N}(\%)$ & $130(58,56)$ \\
\hline \multicolumn{2}{|l|}{ Cantidad de menores de 15 años, $\mathrm{N}(\%)$} \\
\hline 0 & $91(40,9)$ \\
\hline 1 & $59(26,6)$ \\
\hline 2 & $42(18,9)$ \\
\hline 3 & $19(8,6)$ \\
\hline 4 & $8(3,6)$ \\
\hline 5 & $2(0,9)$ \\
\hline 6 & $1(0,5)$ \\
\hline \multicolumn{2}{|l|}{ Escolaridad, $\mathrm{N}(\%)$} \\
\hline Primaria incompleta & $27(12,22)$ \\
\hline Primaria completa & $55(24,89)$ \\
\hline Secundario incompleta & $59(26,70)$ \\
\hline Secundario completo & $70(31,67)$ \\
\hline Terciario incompleto/Universitario incompleto & $4(1,81)$ \\
\hline Terciario completo/Universitario completo & $6(2,71)$ \\
\hline Posgrado & 0 \\
\hline Jefa de hogar mujer, $\mathrm{N}(\%)$ & $101(45,91)$ \\
\hline $\begin{array}{l}\text { Personas que conocen el Centro de Medicina Familiar y } \\
\text { Comunitaria San Pantaleón }\end{array}$ & $179(80,63)$ \\
\hline $\begin{array}{l}\text { Personas que asisten al Centro de Medicina Familiar y } \\
\text { Comunitaria San Pantaleón }\end{array}$ & $108(48,65)$ \\
\hline
\end{tabular}


Se visitaron 505 hogares, entre los cuales 252 (49,9\%) no respondieron luego de dos visitas realizadas en días diferentes y 31 $(6,14 \%)$ personas rechazaron participar. En prácticamente todos los casos la persona que atendió en cada hogar fue la que respondió la encuesta refiriendo que no había, en aquel momento, otras personas mayores de 15 años en el domicilio para responder.
Hubo 150 casos que presentaron scores no extremos y 70 presentaron scores extremos de 0 y 8 .

El análisis de respuesta por ítem se distribuyó de la siguiente forma, ver Tabla 2

Tabla $\mathbf{N}^{\circ} 2:$ : Respuestas afirmativas por ítems

\begin{tabular}{lc}
\hline Escala de Inseguridad Alimentaria basada en la Experiencia & Total=222 \\
Durante los últimos 12 MESES, ha habido algún momento en que: & Ní (\%) \\
\hline $\begin{array}{l}\text { 1- (WORRIED) Usted se haya preocupado por no tener suficientes alimentos para comer por } \\
\text { falta de dinero u otros recursos. }\end{array}$ & $144(64,86)$ \\
$\begin{array}{l}\text { 2- (HEALTHY) Pensando aún en los últimos 12 meses, hubo alguna vez en que usted no } \\
\text { haya podido comer alimentos saludables y nutritivos por falta de dinero u otros recursos. }\end{array}$ & $106(47,75)$ \\
3- (FEWFOOD) Usted haya comido poca variedad de alimentos por falta de dinero u otros \\
recursos \\
$\begin{array}{l}\text { 4- (SKIPPED) Usted haya tenido que dejar de desayunar, almorzar o cenar porque no había } \\
\text { suficiente dinero u otros recursos para obtener alimentos. }\end{array}$ \\
$\begin{array}{l}\text { 5- (ATELESS) Pensando aún en los últimos 12 meses, hubo alguna vez en que usted haya } \\
\text { comido menos de lo que pensaba que debía comer por falta de dinero u otros recursos. }\end{array}$ \\
$\begin{array}{l}\text { 6- (RUNOUT) Su hogar se haya quedado sin alimentos por falta de dinero u otros recursos. } \\
\text { 7- (HUNGRY) Usted haya sentido hambre pero no comió porque no había suficiente dinero u } \\
\text { otros recursos para obtener alimentos. }\end{array}$ \\
$\begin{array}{l}\text { 8- (WHLDAY) Durante los últimos 12 MESES, iha habido algún momento en que usted } \\
\text { haya dejado de comer todo un día por falta de dinero u otros recursos? }\end{array}$ \\
\hline
\end{tabular}

Todos los ítems presentaron valores de Infit y Outfit dentro de los márgenes recomendados (-2 a 2, para los valores estandarizados). Ver Tabla 3.

Tabla $\mathrm{N}^{\circ}$ 3: Comportamiento psicométrico de los ítems

\begin{tabular}{lccc}
\hline & Outfit $^{1}$ & Infit $^{2}$ & Diferencia $^{\mathbf{3}}$ \\
\hline Ítem 1-WORRIED & 1,05 & 1,827 & 0,66 \\
Ítem 2- HEALTHY & 1,324 & 0,683 & 0,54 \\
Ítem 3- FEWFOOD & 0,434 & $-0,582$ & 0,43 \\
Ítem 4- SKIPPED & $-1,566$ & $-1,705$ & 0,21 \\
Ítem 5- ATELESS & $-0,945$ & $-1,428$ & 0,36 \\
Ítem 6- RUNOUT & $-0,175$ & 1,019 & 0,11 \\
Ítem 7- HUNGRY & $-0,884$ & $-1,275$ & 0,04 \\
Ítem 8- WHLDAY & $-0,608$ & $-0,367$ & 0,3 \\
\hline $\begin{array}{l}\text { 1 y 2: Se esperan valores de Outfit e Infit estandarizados que oscilen entre }-2 \text { y 2 (equivalentes a valores estimados de } 0,7 \text { y 1,3 } \\
\text { respectivamente). }\end{array}$ 3 Diferencia absoluta entre la severidad estándar de cada ítem (datos internacionales) luego de la calibración (equiparación de la media \\
y el desvío estándar del conjunto de elementos, en una métrica común), y la severidad del ítem en nuestra muestra.
\end{tabular}

Ninguno de los ítems en el modelo mostró un valor de "p" significativo al test R1c, lo que habría señalado la incongruencia respecto al trazo latente (pendiente común derivada de la contribución de los 8 elementos ${ }^{(18)}$. El valor de "p" del test grupal R1c también fue no significativo $(0,31)$; desplegándose gráficamente la información recolectada en forma regular sobre el intervalo del trazo latente (ver Figura 1) donde los individuos están representados $^{(13)}$. El test $U$ de Molenaar (comparación entre las pendientes de los ítems), mostró valores entre -3 y 3 , para cada elemento, como recomienda la literatura ${ }^{(18)}$. 


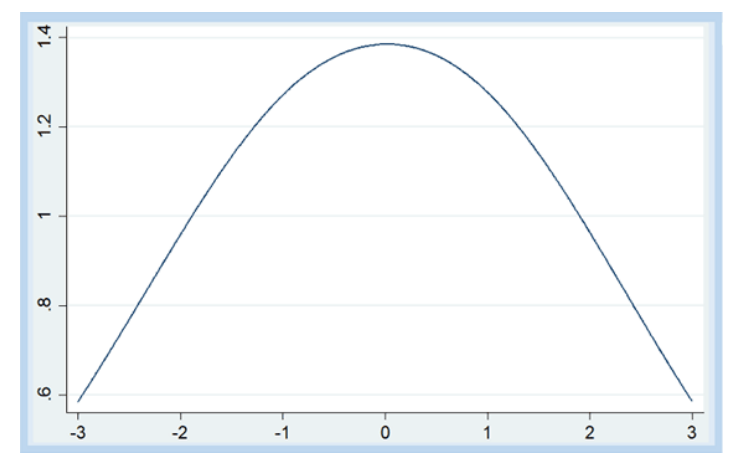

Figura $\mathbf{N}^{\circ} 1$. Información de la escala. Información (en la ordenada, vertical), sobre el trazo latente (en la abscisa, horizontal)

Los valores de diferencia absoluta entre la severidad estándar de cada ítem luego de la calibración, y la severidad del ítem en nuestra muestra se observan también en la Tabla 3. Ningún ítem presentó un valor significativamente diferente por lo tanto todos fueron calificados como "comunes" y la correlación entre ellos fue del $96,43 \%$. La prevalencia de inseguridad alimentaria moderada/severa fue $47,29 \%$ y considerando sólo la severa fue $6,57 \%$.

Calculamos también la prevalencia de inseguridad alimentaria considerando que el ítem Worried tiene una diferencia significativa, y por ende lo calificamos como "único" (Ver Tabla 3). Con esta premisa la correlación entre los ítems comunes se elevó a 97,93 \% y los resultados de inseguridad alimentaria fueron: moderada y severa $=44,74 \%$, y severa $=7,845 \%$

La fiabilidad del modelo (8 ítems) según el Coeficiente KuderRicharson (KR-20) resultó en 0,8727 y según el valor de Fiabilidad compuesta fue de 0,93.

Con el objetivo de determinar variables asociadas a la inseguridad alimentaria se realizó un modelo de regresión logística considerando inseguridad moderada/severa aquellos individuos que respondieron afirmativamente a 4 preguntas o más. Se excluyeron las personas con respuestas incompletas 0 que hubiesen respondido "no sé" a alguno de los ítems, obteniéndose un total de 220 encuestas. La mediana de respuestas afirmativas fue de 3; RIC $0-3$.

Se realizó un modelo de regresión logística que mostró que la inseguridad moderada/severa se asoció en forma independiente a tener una jefa de hogar de sexo femenino (abuela, madre o hija) OR 2,22; (IC95\% 1,27 a 3,87) y con tener más de 4 convivientes en el hogar OR 2,24; (IC95\% 1,25 a 4,02).

\section{DISCUSIÓN}

Se han utilizado diferentes instrumentos para medir la inseguridad alimentaria, tales como el Módulo de encuesta de seguridad (US HFSSM) $)^{(19,20)}$ y ELCSA (Escala Latinoamericana y Caribeña de Seguridad Alimentaria)(21); que han probado ser confiables en diversos contextos culturales. Siguiendo estos lineamientos FAO desarrolló la escala FIES $^{(22)}$, para medir la experiencia directa de las personas sobre la inseguridad alimentaria a nivel individual a escala global $^{(23)}$.

A través de este estudio hemos realizado la validación provisional de la encuesta FIES siguiendo el modelo Rasch propuesto por FAO. Todos los ítems presentaron valores de Infit y Outfit adecuados, los test (ni individuales ni grupales) no mostraron valores significativos para ninguno de los elementos, corroborándose que todos tuvieron un ajuste adecuado. Lo mismo se pudo observar, gráficamente, respecto de la normalidad de la distribución de la información sobre el trazo latente, verificándose la adaptación de la escala para la población estudiada.

La metodología descripta permite obtener datos comparables, ya que se consigue calibrar las escalas de diferentes países en una métrica común al equiparar la media y el desvío estándar del conjunto de elementos ${ }^{(10)}$.
El estudio de Garzón- Orjuela y col. ha realizado la comparación psicométrica del módulo de la escala FIES en tres países latinoamericanos (Colombia, México y Guatemala) concluyendo que presenta las características psicométricas correspondientes al constructo teórico de la herramienta ${ }^{(24)}$.

El reciente informe de FAO indica que en Argentina la inseguridad alimentaria moderada/severa creció del 19,2\% (2014-2016) al $35,8 \%(2017-2019)$ y la inseguridad alimentaria severa del $5,8 \%$ (2014-2016) al 12,9\% (2017-2019) mostrando un aumento progresivo en los últimos años ${ }^{(25)}$. Hallamos una prevalencia de inseguridad alimentaria moderada/severa de $47,29 \%$, mayor a lo que reportan otras fuentes como el Observatorio de la Deuda Social Argentina, quien la define como la reducción involuntaria de la porción de comida o la percepción de experiencias de hambre por problemas económicos durante los últimos doce meses y estima una inseguridad alimentaria total para la población de $22,2 \%{ }^{(26)}$. En relación a la inseguridad severa el porcentaje fue menor $(6,57 \%)$ en comparación con lo publicado por FAO y por la UCA $(9,4 \%)^{(25,26)}$. Esta muestra de la población del Gran Buenos Aires donde según estadísticas recientes (27) hay un de $31,8 \%$ de hogares bajo la línea de la pobreza, ha presentado un alta de prevalencia de inseguridad alimentaria, en concordancia con nuestra percepción inicial que motivó la realización del estudio.

Se encontró una asociación independiente entre la inseguridad alimentaria moderada/severa y las viviendas con mayor cantidad de convivientes y aquellos hogares con jefatura de hogar femenina. Los hogares con mayores convivientes presentan mayor riesgo de inseguridad alimentaria tal como lo muestran estudios realizados en contextos diversos, como el de Wambogo y col. en África subsahariana o el de Costa y col. en Brasil ${ }^{(28,29)}$. Esto podría asociarse a que familias con mayor cantidad de integrantes necesiten más recursos para adquirir alimentos y, especialmente en estos grupos vulnerables, casi nunca aumentan en igual proporción que sus ingresos.

Un punto a destacar es la fuerte asociación entre la mayor inseguridad alimentaria y los hogares encabezados por mujeres, esto también se observó en otros estudios(29-31). Esto deja al descubierto, una vez más, las inequidades de género, y podría sugerir la presencia de desigualdades en el mercado laboral para las mujeres quienes accederían a puestos de trabajo peor remunerados e incluso en iguales condiciones laborales a salarios más bajos ${ }^{(32)}$. Estudios realizados en África, Asia y América Latina, mostraron que las mejoras en materia de seguridad alimentaria y nutrición de las familias están asociadas al acceso de las mujeres al ingreso y el papel que desempeñan en las decisiones que se toman en el hogar sobre el reparto de los gastos, ya que, más que el hombre, la mujer tiende a destinar un porcentaje mucho más elevado de sus ingresos a alimentar a la familia ${ }^{(33)}$. Por ellos es fundamental que las políticas públicas que aborden la seguridad alimentaria tengan un enfoque de género.

Dentro de las limitaciones 0 debilidades de este trabajo mencionamos que la tasa de respuesta fue $44 \%$; si bien no existe un límite exacto para considerarla adecuada, cuanto más nos alejamos del $100 \%$ ideal, más incertidumbre tendremos sobre la validez de los resultados ya que desconocemos la opinión de aquellos que no respondieron. Cada hogar fue visitado dos veces, en diferentes días de la semana y horarios, disminuyendo las probabilidades de un sesgo estructurado y por ende de una variación sistemática en los resultados. En segundo lugar, si bien desde lo metodológico se propuso una representatividad adecuada en cada hogar, a través de una aleatorización de los individuos presentes y mayores de 15 años, las personas que atendían en las diferentes casas, fueron en su mayoría, las únicas disponibles para responder la encuesta lo que podría afectar la representatividad de los resultados.

Dentro de las fortalezas mencionamos que se aplicó una herramienta ampliamente utilizada, y que además fue validada en nuestra muestra a través del testeo de las asunciones del modelo Rasch con excelentes resultados, siguiendo los requerimientos metodológicos ya comentados para poder realizar comparaciones válidas entre mediciones locales e internacionales. 


\section{CONCLUSIONES}

Hasta donde conocemos, sería la primera experiencia en Argentina que utiliza esta herramienta a través de encuestas realizadas personalmente, y no en forma telefónica. Se trataría de un instrumento válido y de sencilla aplicación por lo que sería importante incluirlo en encuestas nacionales que puedan aplicarse en todo nuestro país.

Conocer la prevalencia de inseguridad alimentaria en nuestra población permitirá establecer una comparativa con los valores nacionales y proveer una línea de base para futuras iniciativas. Además constituye un insumo fundamental para las autoridades estatales al momento de formular 0 reformular políticas públicas. En relación con su utilización nos brindará nuevos elementos a considerar como factores intervinientes de las estadísticas epidemiológicas de nuestro centro de salud y orientará las acciones que podremos emprender con la comunidad a fin de mejorar su calidad de vida.

Por último, mencionamos que este trabajo se llevó a cabo antes de la pandemia por COVID-19; sería interesante replicar esta metodología, en los meses posteriores, para determinar el impacto sobre la inseguridad alimentaria.

\section{Agradecimientos:}

Agradecemos a Daniela Corvalan y Lic. Cecilia Bruno por su colaboración en la recolección de datos y al Dr. Sergio Terrasa por su valiosa ayuda y aportes en la revisión del protocolo y del presente manuscrito.

Agradecemos a la Fundación MF que financió el proyecto.

\section{Limitaciones de responsabilidad:}

La responsabilidad de este trabajo es exclusivamente de los autores.

\section{Conflicto de interés:}

Ninguno

\section{Fuentes de apoyo:}

La presente investigación fue realizada con el soporte de la Fundación MF.

\section{Originalidad:}

Este artículo es original y no ha sido enviado para su publicación a otro medio de difusión científica en forma completa ni parcialmente.

\section{Cesión de derechos:}

Los participantes de este trabajo ceden el derecho de autor a la Universidad Nacional de Córdoba para publicar en la RFCM y realizar las traducciones necesarias.

\section{Contribucion de los autores:}

Todos los autores han participado en la concepción del diseño, recolección de la información y elaboración del manuscrito, haciéndose públicamente responsables de su contenido y aprobando su versión final.

\section{BIBLIOGRAFÍA}

1. Organización de las Naciones Unidas para la Agricultura y la Alimentación (FAO). El derecho humano a la alimentación. Roma, 2007. Disponible en: https://www.fao.org/3/a1601s/a1601s.pdf

2. Organización de las Naciones Unidas para la Agricultura y la Alimentación (FAO). Declaración de la Cumbre Mundial Sobre la Seguridad Alimentaria. Cumbre Mundial sobre la Seguridad Alimentaria. Roma, 2009. Disponible en: https://reliefweb.int/sites/reliefweb.int/files/resources/4A2BA55CAA5 00C0B49257682001FOFBB-Informe completo.pdf

3. Bulgach G. ¿Qué entendemos por inseguridad alimentaria? La impactante situación en Argentina. Evidencia, actualización en la práctica ambulatoria. 2019;22(2):e002010. doi: 10.51987/evidencia.v22i2.4222.

4. Diaz DN, Goldberg AS, Fernandez R. Dimensiones de la Seguridad Alimentaria en el nuevo escenario global: ¿el mito del plato vacío? Evolución de la disponibilidad de alimentos per cápita en Argentina y en el mundo entre 1963 y 2013. Buenos Aires: Instituto de Estudios Sociales, CICPES. 2017. Disponible en: http://hdl.handle.net/20.500.12123/8614

5. Organización de las Naciones Unidas para la Agricultura y la Alimentación (FAO). Fondo Internacional de Desarrollo Agrícola (FIDA). Organización Mundial de la Salud (OMS). Programa Mundial de Alimentos (PMA). Fondo de las Naciones Unidas para la Infancia (UNICEF). El estado de la seguridad alimentaria y la nutrición en el mundo 2019. Protegerse frente a la desaceleración y el debilitamiento de la economía. Roma, FAO. 2019. Disponible en: https://reliefweb.int/sites/reliefweb.int/files/resources/ca5162es.pdf

6. Rosso MA, Wicky MI, Nessier MC, Meyer R. Inseguridad alimentaria en la ciudad de Santa Fe: percepción de los ciudadanos. Salud Colectiva. 2015;11(2):234-45. Disponible en: https://www.scielosp.org/pdf/scol/2015.v11n2/235-245/es

7. Salvia A, Tuñón I, Musante B. La inseguridad alimentaria en la Argentina. Hogares Urbanos. Año 2011. Argentina. Observatorio de la Deuda Social Argentina. UCA. 2012. Diponible en: http://wadmin.uca.edu.ar/public/ckeditor/Informe Inseguridad Alime ntaria doc de trabajo .pdf

8. Organización de las Naciones Unidas para la Agricultura y la Alimentación (FAO). The Food Insecurity Experience Scale: Measuring food insecurity through people's experiences. 2017. Disponible en: https://www.fao.org/3/i7835e/i7835e.pdf

9. Organización de las Naciones Unidas para la Agricultura y la Alimentación (FAO). Implementing the Food Insecurity Experience Scale (FIES) in surveys. FAO; 2019. Disponible en: https://www.fao.org/3/ca1454en/CA1454EN.pdf

10. Organización de las Naciones Unidas para la Agricultura y la Alimentación (FAO). Curso: Indicador 2.1.2 de los ODS - Uso de la escala de experiencia de inseguridad alimentaria (FIES) desconocido: ONU. FAO, 2018. Dsiponibe en: https://elearning.fao.org/course/view.php?id=441

11. Organización de las Naciones Unidas para la Agricultura y la Alimentación (FAO). Métodos para la estimación de índices comparables de prevalencia de la inseguridad alimentaria experimentada por adultos en todo el mundo. FAO, 2016. Disponible en: https://www.fao.org/3/i4830s/i4830s.pdf

12. Gallup. How Does the Gallup World Poll Work?: Measures the Attitudes and Behaviors of the World's Residents. Disponible en: https://www.gallup.com/178667/gallup-world-poll-work.aspx

13. Hardouin J-B. Rasch analysis: Estimation and tests with raschtest. Stata Journal. 2007;7(1):22-44. doi: 10.1177/1536867X0700700102

14. Gonzalez Montesinos MJ. El Análisis de Reactivos con el Modelo Rasch Manual Técnico A. Serie: Medición y Metodología. México: Instituto Nacional para la evaluación de la educación. Universidad de Sonora. 2008. Disponible en: https://xdoc. $m x /$ preview/seleccin-de-reactivos-5ec44cd06347a

15 Organización de las Naciones Unidas para la Agricultura y la Alimentación (FAO). Voices of the Hungry. Usando la FIES. Disponible en: $\quad$ http://www.fao.org/in-action/voices-of-thehungry/usando-la-fies/es/

16. Ministerio de Salud de la Provincia de Buenos Aires. Aspectos éticos de la investigación en seres humanos. Buenos Aires. 2016. Disponible en:

https://www.argentina.gob.ar/sites/default/files/ley11044-prov-bsas.pdf

17. República Argentina. Ministerio de Salud. Guía para Investigaciones con Seres Humanos. Objetivos. Argentina. Resolución 1480 / 2011. Dsiponible en: https://www.argentina.gob.ar/normativa/nacional/resoluci\%C3\%B3n1480-2011-187206 
18. Kakwani N, Silber J. Quantitative Approaches to Multidimensional Poverty Measurement. New York: Hampshire Palgrave Macmillan; 2008. doi: 10.1057/9780230582354.

19. Carlson SJ, Andrews MS, Bickel GW. Measuring food insecurity and hunger in the United States: development of a national benchmark measure and prevalence estimates. J Nutr. 1999 Feb;129(2S Suppl):510S-516S. doi: 10.1093/jn/129.2.510S.

20. Coleman-Jensen A, Nord M. Food Insecurity Among Households with Working-Age Adults with Disabilities. Economic Research Report No. (ERR-144). USDA-ERS; 2013. Dsiponible en: https://www.ers.usda.gov/webdocs/publications/45038/34589 err 1 44.pdf? $v=5686.6$

21. Organización de las Naciones Unidas para la Agricultura y la Alimentación (FAO). Escala Latinoamericana y Caribeña de Seguridad Alimentaria (ELCSA): Manual de uso y aplicación. 2012. Disponible en: https://www.fao.org/3/i3065s/i3065s.pdf

22. Ballard T, Kepple A, Cafiero C. The Food Insecurity Experience Scale: Development of a Global Standardfor Monitoring Hunger Worldwide. Roma: Food and Agriculture Organization of the United Nations; 2013. Disponible en: https://www.fao.org/fileadmin/user upload/esa/docs/FIES Technical Paper new\%20and\%20final\%20062014.pdf

23. Smith MD, Kassa W, Winters $P$. Assessing food insecurity in Latin America and the Caribbean using FAO's Food Insecurity Experience Scale. Food Policy. 2017;71:48-61. doi: 10.1016/j.foodpol.2017.07.005.

24. Garzón-Orjuela N, Melgar-Quiñonez H, Eslava-Schmalbach J. Escala Basada en la Experiencia de Inseguridad Alimentaria (FIES) en Colombia, Guatemala y México. Salud Pública Méx. 2018 SepOc;60(5):10. doi: 10.21149/9051.

25. Organización de las Naciones Unidas para la Agricultura y la Alimentación (FAO). Fondo Internacional de Desarrollo Agrícola (FIDA), Organización Mundial de la Salud (OMS), Programa Mundial de Alimentos (PMA), Fondo de las Naciones Unidas para la Infancia (UNICEF). The State of Food Security and Nutrition in the World 2020: Transforming food systems for affordable healthy diets. Roma: Food and Agriculture Organization of the United Nations; 2020. Disponible en: https://www.unicef.org/media/72676/file/SOFI2020-full-report.pdf

26. Bonfiglio Jl. La pobreza más allá de los ingresos nuevo informe sobre pobreza multidimensional 2010-2019: Introducción de datos fundados en un Enfoque de Derechos. Buenos Aires: Observatorio de la Deuda Social Argentina. Universidad Católica Argentina; 2020. Disponible en: https://repositorio.uca.edu.ar/handle/123456789/9524

27. Dirección Provincial de Estadística. Ministerio de Hacienda y Finanzas. Gobierno de la Provincia de Buenos Aires. Incidencia de la pobreza y la indigencia: total 6 aglomerados Provincia de Buenos Aires. Buenos Aires: Dirección Provincial de Estadística; 2020.

28. Wambogo EA, Ghattas H, Leonard KL, Sahyoun NR. Validity of the Food Insecurity Experience Scale for Use in Sub-Saharan Africa and Characteristics of Food-Insecure Individuals. Curr Dev Nutr. 2018 Jul 20;2(9):nzy062. doi: 10.1093/cdn/nzy062.

29. Costa NS, Santos MO, Carvalho CPO, Assunção ML, Ferreira HS. Prevalence and Factors Associated with Food Insecurity in the Context of the Economic Crisis in Brazil. Curr Dev Nutr. 2017 Sep 7;1(10):e000869. doi: 10.3945/cdn.117.000869.

30. Anschau FR, Matsuo T, Segall-Corrêa AM. Insegurança alimentar entre beneficiários de programas de transferência de renda. Rev Nutri. 2012 Abr; 25(2):177-89. doi: 10.1590/S141552732012000200001.

31. Santos JVd, Gigante DP, Domingues MR. Prevalência de insegurança alimentar em Pelotas, Rio Grande do Sul, Brasil, e estado nutricional de indivíduos que vivem nessa condição. Cad. Saúde Pública. 2010 Jan; 26(1):41-9. doi: 10.1590/S0102$311 \times 2010000100005$.
32. Monteiro F, Schmidt ST, Costa IBd, Almeida CCB, Matuda NdS. Bolsa Família: insegurança alimentar e nutricional de crianças menores de cinco anos. Ciênc. saúde coletiva. 2014;19(5):1347-58. doi: 10.1590/1413-81232014195.21462013.

33. Organización de las Naciones Unidas para la Agricultura y la Alimentación (FAO). La mujer y la seguridad. Disponible en: http://www.fao.org/focus/s/women/Sustin-s.htm. 